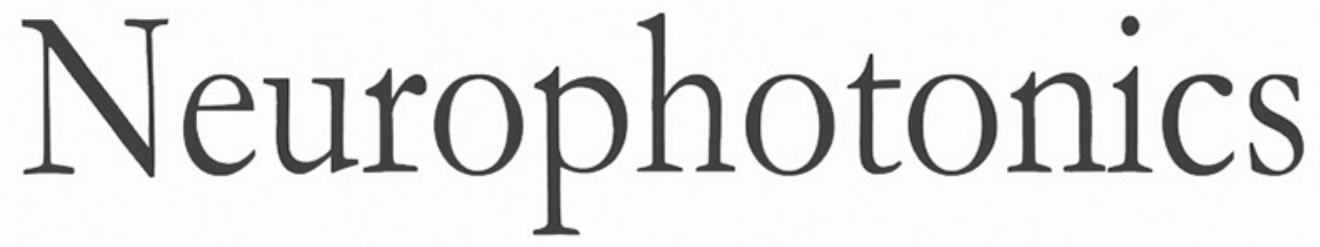

Neurophotonics.SPIEDigitalLibrary.org

\title{
2019 List of Reviewers
}


Neurophotonics would like to sincerely thank the following individuals who served as reviewers in 2019. The success of our publication hinges on the voluntary contributions of time and energy put forth by these professionals.

Yama Akbari
Anna Letizia Allegra Mascaro
Maria Arredondo
David Atchison
Jaideep Bains
Wesley Baker
Matilde Balbi
Gemma Bale
Adam Bauer
Bernhard Baumann
Lisa Beckman
Lisa Beckmann
Ken Berglund
Jason Berwick
Irving Bigio
Pablo Blinder
David Boas
Heather Bortfeld
Sabrina Brigadoi
Ralf Brinkmann
Erin Buckley
Stefan Carp
Paolo Cassano
Siyu Chen
Xiaojun Cheng
Mykyta Chernov
Carol Y. Cheung
Antonio Chiarelli
Adam Cohen
Emma Condy
Irene Costantini
Verginia Cuzon Carlson
Jerry Dadap
Irene de la Cruz Pavia
Hamid Dehghani
Anna Devor
Ilka Diester
Patrick Drew
Adam Eggebrecht
Christian Eggeling
Rinat Esenaliev
Zoltan Fekete
Rodrigo Forti
Dan Fu
Tsukasa Funane
Tamas Fuzesi
Jessica Gemignani
Michael Giacomelli Goto
Danielle Harper
Joy Hirsch
Fumitaka Homae
Keum-Shik Hong
Jue Hou

Yama Akbari

Maria Arredondo

David Atchison

Gemma Bale

Lisa Beckman

Lisa Beckmann

Heather Bortfeld

Sabrina Brigado

Stefan Carp

Paolo Cassano

Siyu Chen

Xiaojun Cheng

Mykyta Chernov

Adam Cohen

Emma Condy

Verginia Cuzon Carlson

erry Dadap

Anna Devor

ka Diester

Dan Fu

Tsukasa Funane

Tamas Fuzesi

Jessica Gemignan

Michael Giacomelli

Greg Giannone

Ariel Gilad

Yukiori Goto

Michael Hamblin

Joy Hirsch

Keum-Shik Hong

Jue Hou

\author{
Zhihong $\mathrm{Hu}$ \\ Xiangrun Huang \\ Daniel Huber \\ Jennifer Hunter \\ E. Duco Jansen \\ Huabei Jiang \\ Kivilcim Kilic \\ Beop-Min Kim \\ Chulhong Kim \\ Joseph La Manna \\ Hsinyi Lai \\ Frederic Lange \\ Kirill Larin \\ Francesco LaRocca \\ Frederic Lesage \\ Lei Li \\ Peng Li \\ Adam Liebert \\ David Liebeskind \\ Chao Liu \\ Hanli Liu \\ Ting Luo \\ Hongtao Ma \\ Nicholas MacKinnon \\ Ryan McNabb \\ Yukifumi Monden \\ Christopher Moore \\ Yusuke Moriguchi \\ Timothy Murphy \\ Noman Naseer \\ Kamran Nasiriavanaki \\ Thien Nguyen \\ Ruiqing $\mathrm{Ni}$ \\ Konstantin Nikolic \\ Haijing Niu \\ Sergio Novi Junior \\ Jonathan Nylk \\ Rodney O'Connor \\ Marco Pagliazzi \\ B. Hyle Park \\ Ashwin Parthasarathy \\ Stephane Perrey \\ Shaohua Pi \\ Daqing Piao \\ Paola Pinti \\ Laura Pirazzoli \\ Filippo Pisano \\ Philippe Pouliot \\ Jun Qian \\ Ajay Rajaram \\ Anna Roe \\ John Rogers \\ Caroline Runyan \\ Sava Sakadzic \\ Michelle Sander \\ Hendrik Santosa \\ Hiroki Sato
}

Christopher Schaffer

Felix Scholkmann

Fei Shi

Andy Shih

Pavel Shilyagin

Sotaro Shimada

Shy Shoham

Alejandra Sierra Lopez

Ludovico Silvestri

Garth Simpson

Weiye Song

John Spencer

Vivek Srinivasan

Colin Sullender

Jason Sutin

Stephanie Sutoko

Nick Swindale

Ilias Tachtsidis

Toshimitsu Takahashi

Stefan Talu

Johnny Tam

Jianbo Tang

Qinggong Tang

Martin Thunemann

Jan Tonnesen

Peter Torok

Antoine Triller

Kotaro Tsuboi

Daisuke Tsuzuki

Vassiliy Tsytsarev

Ben Urban

Matt Valley

Gregoire Vergotte

Alexander von Lühmann

Heidrun Wabnitz

Zheng Wang

Jack Waters

Timothy Weber

Wei Wei

Kit Werley

Lara Wierenga

Ian Winship

Wang Xi

Augix Xu

Guohua Xu

Ninglong Xu

Junjie Yao

Xincheng Yao

Mohammad Yaseen

Ji Yi

Zhen Yuan

Pengfei Zhang

Tingwei Zhang

Wen Zhang

Xian Zhang

Chaozhe Zhu

Bernhard Zimmermann 